\title{
Flame Self-Interactions with Increasing Turbulence Intensity
}

\author{
S. Trivedi*, G.V. Nivarti*, R.S. Cant* \\ Engineering Department, University of Cambridge, Trumpington Street, Cambridge, CB2 \\ $1 P Z, U K$
}

\begin{abstract}
The topology of flame-flame interaction is analysed for single turbulent premixed flames with increasing turbulence intensity. Morse theory for critical points is used for identifying the flame-flame interaction and characterising the local topology. The interactions have been categorised into four different groups, namely reactant pocket, tunnel formation, tunnel closure and product pocket. A histogram showing the frequency of occurrence of each of these groups is presented for single flames representative of hydrocarbon-air combustion and is compared with the results of colliding hydrogen-air flames. It is observed that most interactions for a single flame occur toward the leading edge. Also, more interactions are observed for higher intensity turbulence. The cylindrical topology types are found to dominate over spherical topology types. The relative frequency of occurrence of each type of topology is observed to change with changes in turbulence intensity. With increasing turbulence intensity, the fraction of product pockets and tunnel formation events increases whereas the fraction of reactant pockets and tunnel closure
\end{abstract}

\footnotetext{
${ }^{*}$ Corresponding author:

Email address: st634@cam.ac.uk (S. Trivedi) 
events decreases. The rise in product pockets is mirrored by the drop in reactant pockets, and likewise, the rise in tunnel formation events is mirrored by the drop in tunnel closure events.

Keywords:

Turbulent Combustion, DNS, Flame-flame interaction, Flame topology, Morse Theory

\section{Introduction}

A flame surface on interaction with a turbulent flow becomes wrinkled, resulting in increased flame surface area [1]. This increase in area gives rise to increased flame propagation speeds. However, when the flame is highly wrinkled, the interaction of a flame with itself locally is inevitable [2-5], particularly in high intensity turbulence [6-8]. The local topology of flame-flame interactions governs phenomena such as cusp formation or pocket burnout, which can significantly alter the flame surface area and therefore affect the burning rate [9-14]. A fundamental understanding of these processes is required in order to analyse their effect on the overall surface area of a flame and incorporate these findings into mathematical models.

Flame surface can be defined by an iso-surface of a reaction progress variable provided that flamelet assumption is valid [15]. To quantify the flame-flame interaction, Morse theory [16] of critical points has been used by Chen et al. [10] and Griffiths et al. [17]. The thoery indicates that during a flame-flame interaction the two flame surfaces will mutually annihilate each other [11]. At such a point of contact, the gradient of the reaction progress variable is equal to zero. This point is called a critical point. In the vicinity 
of this point, the Hessian of the progress variable will contain information on local topology. Based on the eigenvalues of the Hessian, the local shape factors can be evaluated and the local topology can be described [17].

A two-dimensional Direct Numerical Simulation (DNS) study for a lean methane-air premixed flame by Chen et al. [10] was able to explain the mechanism of pocket formation. A three-dimensional study then followed, emphasising the flame-flame interaction for a pair of colliding hydrogen-air flames [17] using the DNS data of Hawkes et al. [18]. Using the shape factors, Griffiths et al. [17] defined all possible topologies of flame-flame interactions and categorised them into four main groups, namely "reactant pocket" (RP), "tunnel closure" (TC), "tunnel formation" (TF) and "product pocket" (PP). This can be seen in Fig. 1. The frequency of occurrence of each of the possible topologies was then evaluated [17].

This analysis was done for two counter-propagating hydrogen-air flames. However, flame-flame interactions exist even within a single flame and can cause changes in flame propagation behaviour. A single-flame dataset is required to quantify the self interactions in order to better understand their impact on flame surface area and propagation speed. In this paper, the three-dimensional DNS dataset of Nivarti et al. [22] for single premixed turbulent flames is used to investigate flame-flame interaction topologies using the numerical tools of Griffiths et al. [17].

The next section of this paper describes the mathematical background of the Morse theory for critical points. Section 3 describes the dataset by Nivarti et al. [22] that is used for the current study. The results obtained for this dataset are discussed in section 4. In particular, histograms are created 


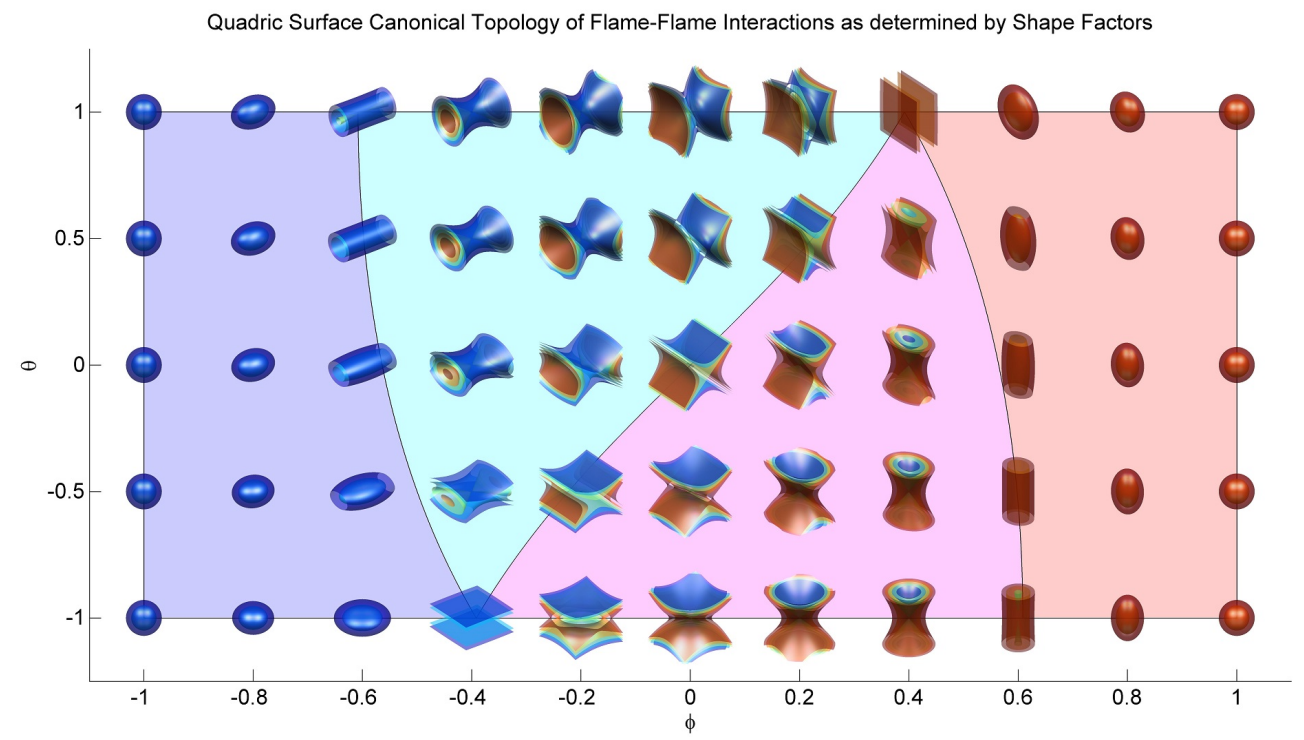

Figure 1: The set of all possible flame-flame interaction topologies as determined by the shape factors [17].

for the single flame dataset for direct comparison with the previous twoflame hydrogen data. Results are also presented to show the change in the relative frequencies of occurrence of each topology as the turbulence intensity is increased.

\section{Topology of flame-flame interaction}

The changes in premixed flame propagation behaviour due to flame-flame interaction can be quantified using Morse theory [16]. The mathematical basis of these tools is discussed below, followed by a discussion on the results obtained by Griffiths et al. [17].

For flames that are not in the broken reaction zone regime, a reaction progress variable $c$ can be used to characterise the flame. A definition of $c$ 
using a suitable choice of species mass fraction is

$$
c=\frac{Y_{\alpha}-Y_{\alpha R}}{Y_{\alpha P}-Y_{\alpha R}}
$$

The value of $c$ goes monotonically from zero in the reactants to unity in the products. An iso-surface of $c$ can be used to define a flame surface. A critical point is defined as a point where the gradient of the progress variable is equal to zero. Critical points are found where two flame fronts collide [17].

The appearance of critical points provides a means to describe the flameflame interaction using Morse theory [16]. The Taylor expansion of the progress variable around a critical point where the gradient is zero is given by

$$
c(a+x)=c(a)+\frac{x^{T}}{2} H(c(a)) x+\ldots
$$

The Hessian function $H(c)$ contains information on the local topology in the vicinity of the critical point. The eigenvalues of the Hessian $\lambda_{1}, \lambda_{2}$ and $\lambda_{3}$ give the curvature along the three orthogonal principal axes. Shape factors $\theta$ and $\phi$ and mean curvature $\kappa$ can be derived using the eigenvalues of the Hessian according to

$$
\begin{gathered}
\theta=\frac{6}{\pi} \arctan \frac{\left(\lambda_{1}-2 \lambda_{2}+\lambda_{3}\right) / 6^{1 / 2}}{\left(\lambda_{1}-\lambda_{3}\right) / 2^{1 / 2}} \\
\phi=\frac{2}{\pi} \arctan \frac{\left(\lambda_{1}+\lambda_{2}+\lambda_{3}\right) \cos (\theta \pi / 6) / 3^{1 / 2}}{\left(\lambda_{1}-\lambda_{3}\right) / 2^{1 / 2}} \\
\kappa=\left(\lambda_{1}^{2}+\lambda_{2}^{2}+\lambda_{3}^{2}\right)^{1 / 2}
\end{gathered}
$$


The shape factors fully define the local topology of the flame-flame interactions. Griffiths et al. [17] determined the full set of all possible flame-flame interaction topologies based on the shape factors (Fig. 1). These topologies included four particular cases named "product pockets", "tunnel closure", "tunnel formation" and "reactant pockets". Tunnel closure and tunnel formation are cylindrical topologies while the reactant and product pockets are spherical topologies.

In the two-dimensional flame topology study by Chen et al. [10, 11], the mechanism for pocket formation for a lean methane-air flame was investigated. The pocket formation was shown to occur in three stages: (1) merging of the reaction rate of flames at very short timescales, (2) cusp recovery and (3) formation of an isolated flame pocket which eventually burns out. Another important aspect of this two-dimensional study was that the strain and curvature effects were found to play an important role in pocket formation.

A subsequent 3-D study was performed by Griffiths [17] using data of Hawkes et al. [18]. A progress variable based on the mass fraction of $\mathrm{H}_{2} \mathrm{O}$ was used for tracking the flame. The location of the critical points was evaluated by using the Newton method based on tri-quintic interpolation [19]. A histogram was created showing the frequency of occurrence of different topologies at different locations within the flame. Results showed that cylindrical topologies were dominant over spherical topologies (Fig. 2) which is consistent with the study of Pope et al. [20] and Cant et al. [21] for a laminar flamelet.

These results were obtained for colliding hydrogen-air flames, and it is 


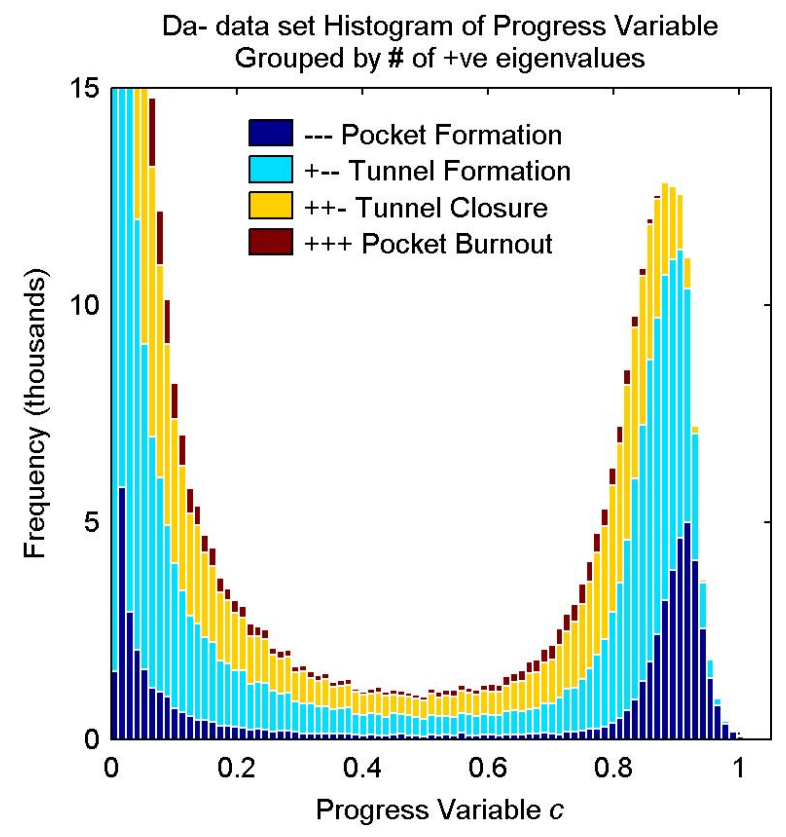

Figure 2: Histogram showing the relative frequency of occurrence of the four principal topologies [17]

useful to investigate whether the findings will change for self-interaction of a single flame or for flames representative of more practical hydrocarbon-air combustion. These questions will be addressed in the following sections.

\section{DNS dataset for the current study}

The DNS data analysed here are part of a published dataset $[22,23]$ generated using the Senga2 solver [25]. This dataset consists of five separate simulations conducted by solving the Navier-Stokes equations in 3D compressible form along with a transport equation for the reacting species. Each case simulates a statistically-planar flame brush propagating freely towards the inlet in an inflow-outflow configuration. The domain is initialised with a 
planar laminar flame surrounded by a field of homogeneous isotropic turbulence of desired intensity computed a priori. A copy of the initial turbulent flow field is also convected through the inlet so as to maintain the turbulence ahead of the flame. The intensity of the turbulent flow field is increased successively across the simulation dataset. Flame chemistry is represented using a single-step Arrhenius reaction mechanism with the Lewis number of the reacting species set to unity.

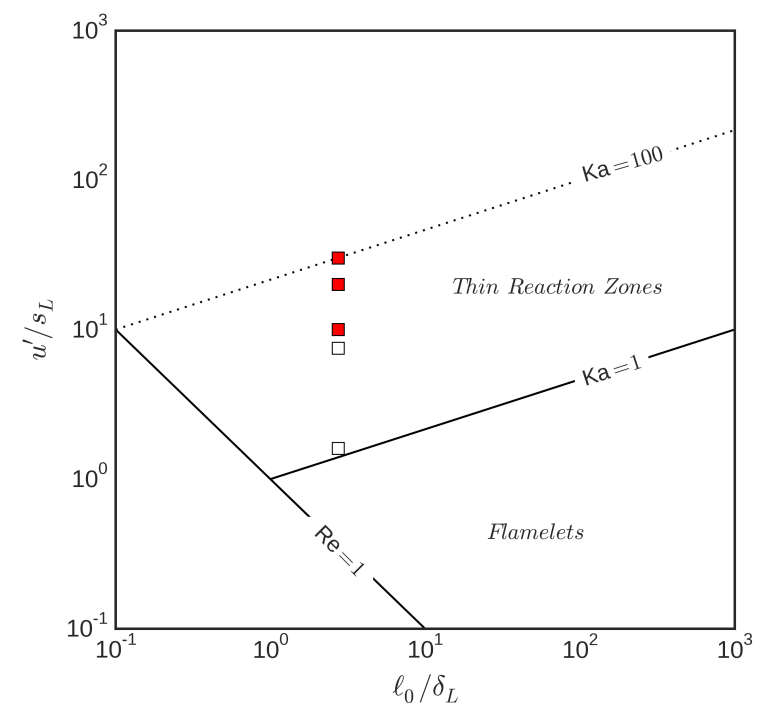

Figure 3: Regimes of combustion in the simulation dataset indicating the cases investigated here (red squares).

The reaction mechanism is tuned to replicate the flame propagation speed of stoichiometric methane-air flame, i.e $39 \mathrm{~cm} / \mathrm{s}$, and also to capture the corresponding thickness of the reaction zone. Furthermore, Nivarti et al. [24] validated the results obtained for a high-intensity case in this dataset using detailed 25-step chemical mechanism for methane-air combustion [26]. Hence the DNS dataset can be taken as representative of hydrocarbon-air 
combustion.

A distinguishing feature of the dataset is that all thermo-chemical and physical parameters are maintained constant with the exception of turbulence intensity $u^{\prime}$. The value of $u^{\prime}$ ranges from as low as $1.5 s_{L}$ to as high as $30 s_{L}$. The Karlovitz number

$$
\mathrm{Ka}=\frac{u^{\prime}}{\lambda} \frac{\delta_{L}}{s_{L}} \sim\left(\frac{u^{\prime}}{s_{L}}\right)^{\frac{3}{2}}\left(\frac{\delta_{L}}{\ell_{0}}\right)^{\frac{1}{2}},
$$

based on the Taylor length scale $\lambda$ varies across the dataset. In Fig. 3, the regime corresponding to the simulations is shown in the Borghi diagram. Further details are provided in the references [22, 23].

\section{Results}

Three of the five simulations in the dataset [22] relevant to the current study were analysed. For lower intensity turbulence, fewer flame-flame interactions were observed so the cases of $u^{\prime}=1.5 s_{L}$ and $u^{\prime}=5 s_{L}$ were discarded. The three cases that were analysed correspond to $u^{\prime}=10 s_{L}, u^{\prime}=20 s_{L}$, and $u^{\prime}=30 s_{L}$, respectively. Each of the cases was run for a time $t=6 \tau_{0}$ eddy turn over times corresponding to each $u^{\prime}$ level.

In the above dataset, no broken reaction zones were observed, and hence a progress variable iso-surface will adequately define a flame surface. A progress variable based on product mass fraction was used for this study, defined according to

$$
c=\frac{Y_{P}}{Y_{P, P r o d}}
$$

The computational tools of Griffiths et al. [17] were applied to this dataset. These tools were adjusted to work for the case of a single flame. 
The resulting histograms obtained in the same way as Fig. 2 are presented in Fig. 4, going from $u^{\prime}=10 s_{L}$ at the top to $u^{\prime}=30 s_{L}$ at the bottom. The histograms on the left are for the leading edge of the flame, that is, $0.01<c<0.2$ and those on the right are for the rest of the range of $c$, that is, $0.2<c<0.99$. Note that the numbers of interactions on the leading edge (left) are of a much higher magnitude than for the rest of the flame (right).

From Fig. 4, it is clear that the Griffiths technique works well for the single flame dataset. All the interactions observed are self interactions within a single flame. Consistent with the previous results [17], more interactions are observed for increasing turbulence intensity going from $u^{\prime} / s_{L}=10$ at the top to $u^{\prime} / s_{L}=30$ at the bottom. Numerous flame-flame interactions take place even within a single flame, particularly at high turbulence intensities. Also, the cylindrical topologies (TF and TC) are dominant over spherical topologies (PP and RP) which is consistent with the findings of Pope et al. [20] and Cant et al. [21].

The reason for flame-flame interactions being dominant in the leading edge is due to dilatation which acts to dissipate most of the turbulence as the temperature increases within the flame. This can be seen in Fig. 5 showing contours of the progress variable at the leading and trailing edges for $u^{\prime} / s_{L}=20$. The flame is highly wrinkled at the leading edge resulting in more interactions, whereas the trailing edge is smoother showing very little self interaction.

Table 1 shows the fraction of each type of topology for increasing turbulent intensities. It can be seen that cylindrical topologies (tunnel formation TF and tunnel closure TC) account for about $80 \%$ of the total number of 

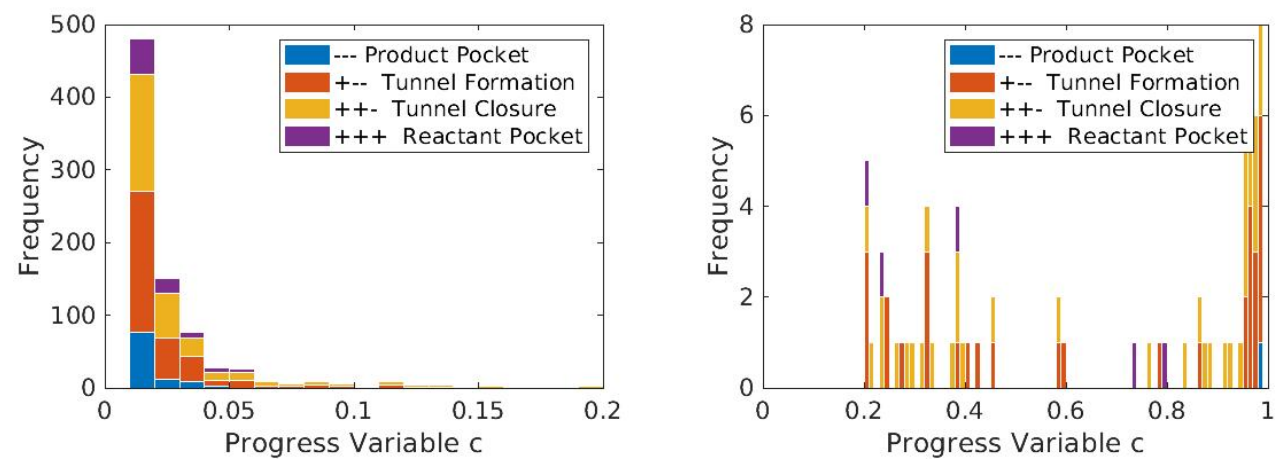

a) $u^{\prime} / s_{L}=10$
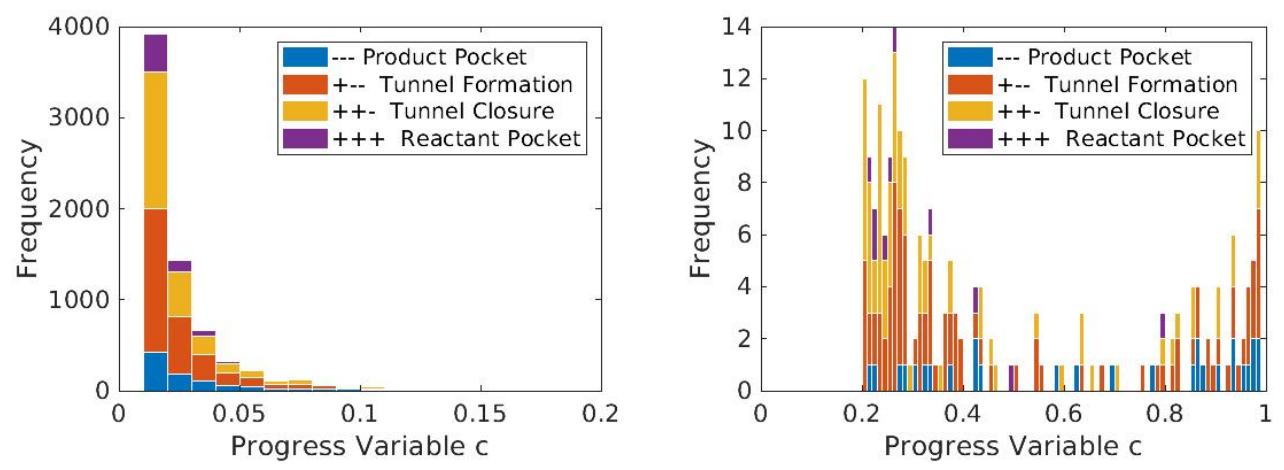

b) $u^{\prime} / s_{L}=20$
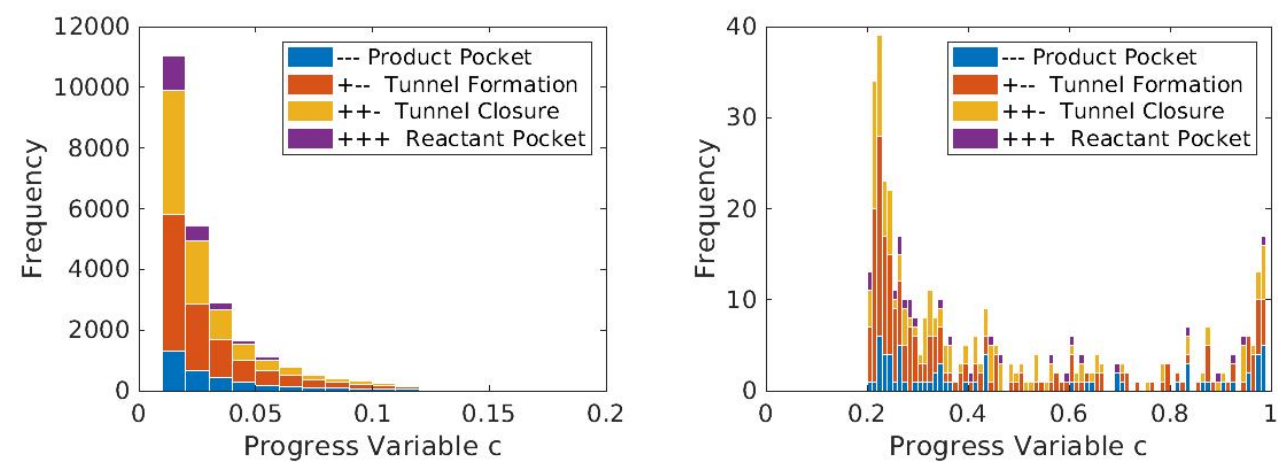

c) $u^{\prime} / s_{L}=30$

Figure 4: Histograms for the frequency of occurrence of different topologies represented by different colours. Histograms on the left represent the leading edge $(0.01<c<0.2)$ and histograms on the right represent the rest of the flame $0.2<c<0.99$ ) 


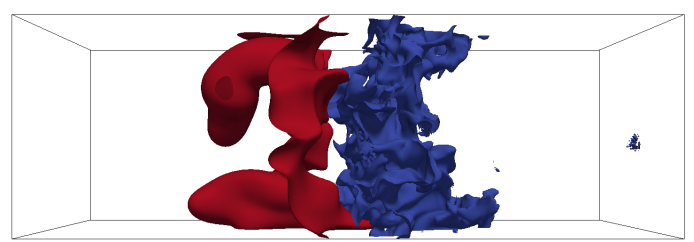

Figure 5: Two different iso-surfaces of the progress variable at $u^{\prime} / s_{L}=20$. Blue surface represents $c=0.02$ and red surface represents $c=0.99$. The difference in wrinkling is apparent

observed interactions while spherical topologies (product pockets PP and reactant pockets RP) account for about $20 \%$ of the total interactions.

\begin{tabular}{|l|l|l|l|l|}
\hline \multicolumn{4}{|c|}{ Topology Fractions for increasing $u^{\prime} / s_{L}$} \\
\hline$u^{\prime} / s_{L}$ & $\mathrm{PP}$ & $\mathrm{TF}$ & $\mathrm{TC}$ & $\mathrm{RP}$ \\
\hline 10 & 0.1130 & 0.394 & 0.381 & 0.112 \\
20 & 0.1280 & 0.4124 & 0.3630 & 0.0965 \\
30 & 0.1345 & 0.4203 & 0.355 & 0.0898 \\
\hline
\end{tabular}

Table 1: Fraction of topologies for increasing turbulent intensities

An interesting picture emerges when plotting the fraction of topologies with increasing turbulence intensity (Fig. 6 top). Going from $u^{\prime} / s_{L}=10$ to 30 , it is observed that proportionately more product pockets are formed within the domain. It is also clearly observed that the rise in the proportion of product pockets is almost mirrored by the drop in the proportion of reactant pockets.

A similar observation is made for tunnel formation and tunnel closure (Fig. 6 bottom), in that there is a steady rise in the proportion of tunnel formation and an almost identical drop in the proportion of tunnel closure. 


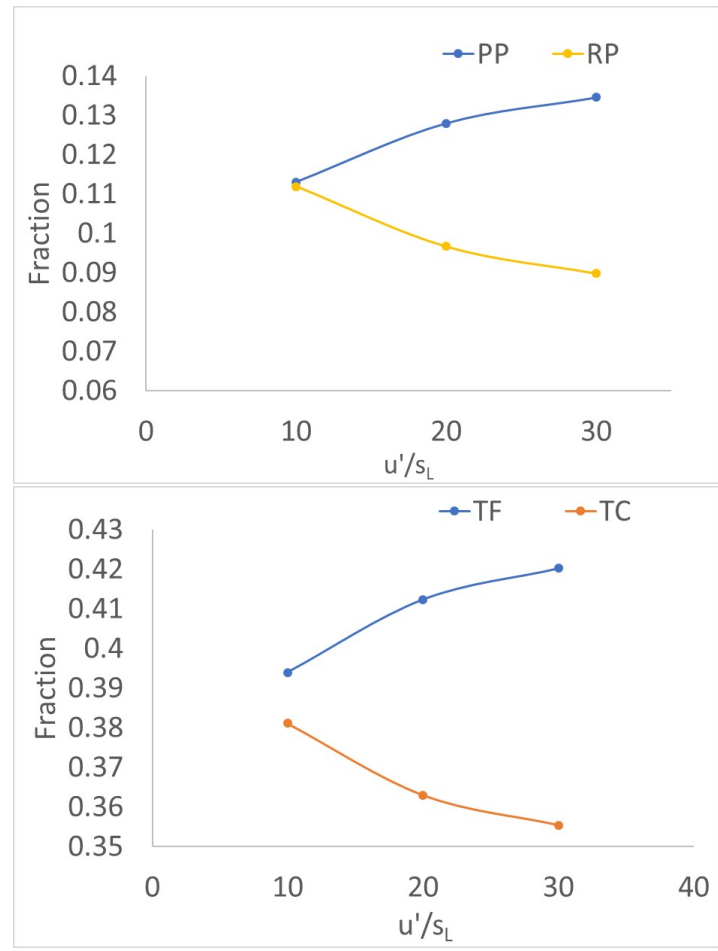

Figure 6: Fractions of Reactant Pockets (RP) and Product Pockets (PP) (top), and fractions of Tunnel Formation (TF) and Tunnel Closure (TC) (bottom)

The effect of each individual topology on flame propagation is still unknown and is being investigated as part of ongoing work. However, the above result may have implications for flame propagation with increasing turbulence intensity. The reactant pocket and tunnel closure topologies result in a very high displacement speed near burnout $[10-12,27]$. The drop in their proportions indicates that the overall flame speed might be reduced at higher intensities. 


\section{Conclusions}

From the above analysis, it is clear that the flame-flame interaction technique developed by Griffiths et al. [17] is also valid for the single flame dataset capturing the self interactions within a flame. The histogram of topologies shows a similar trend to that found by Griffiths et al. [17] in that more interactions are found for higher turbulence intensities. Flame-flame interactions in a single flame are mostly found at the leading edge and are not frequent at the trailing edge.

A significant result is that the proportion of different topologies varies with the turbulence intensity. There is an increase in product pockets and tunnel formation events with increasing turbulent intensity from $u^{\prime} / s_{L}=10$ to $u^{\prime} / s_{L}=30$, and an almost identical drop in reactant pockets and tunnel closure events for those intensities.

In future studies, it will be important to investigate the exact effect of each type of topology in terms of flame propagation and consumption speed. It is known that pocket burnout and tunnel closure are relatively fast events and hence, a drop in their proportion might result in rapid destruction of flame area and reduced flame propagation speed. It will also be interesting in future to compare the numerical results with experimental results for flames in high intensity turbulence [28].

\section{Acknowledgements}

The authors are grateful for the financial support from the Cambridge Trust and the Nehru Trust. Special thanks are due to Ryan Griffiths for providing the numerical tools and for valuable discussions on this topic. 
[1] G. Damköhler, Zeitschrigt für Elektrochemie und angewandte physikalische Chemie 46(11) (1940) 601-652 .

[2] T.D. Dunstan, N. Swaminathan, K.N.C. Bray, N.G. Kingsbury, Combust. Sci. Tech. 185 (2013) 134-159.

[3] R. Borghi, Combust. Flame 80 (1990) 304-312.

[4] A.A. Burluka, A. Gorokhovski, and R. Borghi, Combust. Flame 109 (1997) 173-187.

[5] A.R. Kerstein, Proc. Combust. Inst. 29 (2002) 1763-1773.

[6] N. Chakraborty, R.S. Cant, Combust. Flame 156 (2009) 1427-1444.

[7] D.O. Lignell, J.H. Chen, H.A. Schmutz, Combust. Flame 158 (2011) 949-963.

[8] H. Kolla, R.W. Grout, A. Gruber, J.H. Chen, Combust. Flame 159 (2012) 2755-2766.

[9] S. Candel, D. Durox, and T. Schuller, AIAA J. (2004) 2004-2928.

[10] J.H. Chen, T. Echekki, W. Kollman, Combust. Flame, 116 (1999) 15-48.

[11] T. Echekki, J.H. Chen, I.R. Gran, Proc. Combust. Inst., 26 (1996) 855.

[12] A.Y. Poludnenko, E.S. Oran, Combust. Flame 157 (2010) 995-1011.

[13] A.Y. Poludnenko, E.S. Oran, Combust. Flame 158 (2011) 301-326.

[14] Ya.B. Zeldovich, A.G. Istratov, N.I. Kidin, V.B. Librovich, Combust. Sci. Tech. 24(1980) 1-13. 
[15] N. Peters, Proc. Combust. Inst., 21 (1986) 1231-1250.

[16] J. Milnor, Analysis of Math. Studies, Princeton University Press (1963) p. 51.

[17] R.A.C. Griffiths, J.H. Chen, H. Kolla, R.S. Cant, W. Kollman, Proc. Combust. Inst., 35 (2014) 1341-1348.

[18] E.R. Hawkes, O. Chatakonda, H. Kolla, A.R. Kerstein, J.H. Chen, Combust. Flame 159 (2012) 2690-2703.

[19] W.H. Press, S.A. Teukolsky, W.T. Vetterling, B.P. Flannery, Numerical Recipes, 3rd ed., Cambridge University Press, Cambridge, U.K., 2007.

[20] S.B. Pope, P.K. Yeung, S.S Girimaji, Phys. Fluids A 12 (1989) 20102018.

[21] R.S. Cant, C. Rutland, A. Trouve, Statistics for laminar flamelet modelling, Proceedings of the summer program, Centre for turbulence research, Stanford University/NASA-AMES (1990) p. 299-310.

[22] G.V. Nivarti, R.S. Cant, Proc. Combust. Inst. 36 (2017) 1903-1910.

[23] G.V. Nivarti, R.S. Cant, Phys. Fluids 29 (2017), 085-107.

[24] G.V.Nivarti, R.S.Cant, Premixed flame propagation in high-intensity turbulence: investigating the role of detailed chemistry, $25^{\text {th }}$ Int. Conf. for Dynamics of Explosions and Reactive Systems, 028 (2015).

[25] R.S. Cant, Senga2: User Guide, Technical Report CUED-THERMO2012/04 - 2, Cambridge University Engineering Department. 
[26] V. Giovangigli, M.D. Smooke, in: M.D Smooke (ed), Reduced Kinetic Mechanisms and Asymptotic Approximations for Methane-Air Flames: A Topical Volume, Lecture Notes in Physics 384, Springer-Verlag, Berlin (1991)

[27] N. Peters, P. Terhoeven, J.H. Chen, T. Echekki, Proc. Combust. Inst., 27 (1998) 833-839.

[28] T.M. Wabel, A.W. Skiba, J.F. Driscoll, Proc. Combust. Inst., 36 (2017) 1801-1808. 